\title{
On the well-posedness of the hyperelastic rod equation
}

\author{
Hasan $\operatorname{Inci}^{1,2}$
}

Received: 26 March 2018 / Accepted: 4 October 2018 / Published online: 10 October 2018 (c) Fondazione Annali di Matematica Pura ed Applicata and Springer-Verlag GmbH Germany, part of Springer Nature 2018

\begin{abstract}
In this paper we consider the hyperelastic rod equation on the Sobolev spaces $H^{s}(\mathbb{R}), s>3 / 2$. Using a geometric approach we show that for any $T>0$ the corresponding solution map, $u(0) \mapsto u(T)$, is nowhere locally uniformly continuous. The method applies also to the periodic case $H^{s}(\mathbb{T}), s>3 / 2$.
\end{abstract}

Keywords Hyperelastic rod equation · Solution map · diffeomorphism groups

Mathematics Subject Classification 35Q35

\section{Introduction}

We consider the following family of equations referred to as the hyperelastic rod equation

$$
u_{t}-u_{t x x}+3 u u_{x}=\gamma\left(2 u_{x} u_{x x}+u u_{x x x}\right), \quad t \in \mathbb{R}, x \in \mathbb{R}
$$

where $\gamma \neq 0$. The initial value problem for (1) is locally well-posed in the Sobolev spaces $H^{s}, s>3 / 2$ - see [6,7]. For the corresponding solution map it was shown in [5] in the periodic case that it has not the property to be uniformly continuous on bounded sets, whereas in [3] the same was shown for both cases (periodic and nonperiodic) with an improvement for the $s$ range. Our aim here is to prove that the solution map for the range $s>3 / 2$ has even less regularity. But before we state the main theorem we have to introduce some notation. Note that (1) has the property that for a solution $u$ the scaled quantity

$$
u_{\lambda}:=\lambda u(\lambda t, x), \quad \lambda>0
$$

is also a solution. Let $s>3 / 2$ and $T>0$. Denote by $U_{T}$ the initial values $u_{0} \in H^{s}(\mathbb{R})$ for which (1), starting from $u(0)=u_{0}$, has a solution which exists longer than $T$. By the local well-posedness of (1) in $H^{s}(\mathbb{R})$ (see also Theorem 2.1) and scaling (2) we know that

Hasan Inci

hinci@ku.edu.tr

1 EPFL SB MATHAA PDE, MA C1 627 (Bâtiment MA), Station 8, 1015 Lausanne, Switzerland

2 Present Address: Department of Mathematics, Koç University, Rumelifeneri Yolu, 34450 Sarıyer, İstanbul, Turkey 
$U_{T} \subseteq H^{s}(\mathbb{R})$ is an open star-shaped neighborhood of $0 \in H^{s}(\mathbb{R})$ in $H^{s}(\mathbb{R})$. Again by the local well-posedness we know that the time $T$ solution map

$$
\Phi_{T}: U_{T} \subseteq H^{s}(\mathbb{R}) \rightarrow H^{s}(\mathbb{R}), \quad u_{0} \mapsto u(T)
$$

is continuous. With this our main theorem reads as

Theorem 1.1 Let $s>3 / 2$ and $T>0$. Denote by $\Phi_{T}$ the time $T$ solution map of the initial value problem for (1) defined on $U_{T} \subseteq H^{s}(\mathbb{R})$. Then

$$
\Phi_{T}: U_{T} \rightarrow H^{s}(\mathbb{R}), \quad u(0) \mapsto u(T)
$$

is nowhere locally uniformly continuous.

We will rewrite (1) by doing the transformation $v(t, x)=u(t, \gamma x)$. This gives

$$
v_{t}-\frac{1}{\gamma^{2}} v_{t x x}+\frac{3}{\gamma} v v_{x}=\frac{1}{\gamma^{2}}\left(2 v_{x} v_{x x}+v v_{x x x}\right)
$$

or rewritten

$$
\left(1-\frac{1}{\gamma^{2}} \partial_{x}^{2}\right)\left(v_{t}+v v_{x}\right)=\frac{\gamma-3}{\gamma} v v_{x}-\frac{1}{\gamma^{2}} v_{x} v_{x x}
$$

and equivalently

$$
v_{t}+v v_{x}=\left(1-\frac{1}{\gamma^{2}} \partial_{x}^{2}\right)^{-1}\left(\frac{\gamma-3}{\gamma} v v_{x}-\frac{1}{\gamma^{2}} v_{x} v_{x x}\right)=: B(v, v)
$$

Note that $B$ is a continuous quadratic form on $H^{s}(\mathbb{R})$ for $s>3 / 2$. We will establish Theorem 1.1 by showing the corresponding statement for the solution map of $v$. This is clearly sufficient. The advantage of (3) is that it is convenient for the geometric framework introduced in the next section.

\section{The geometric framework}

We will formulate (3) in a geometric way as was done in [2] for the $b$-family of equations. Consider the flow map of $v$, i.e.,

$$
\varphi_{t}(t, x)=v(t, \varphi(t, x)), \quad \varphi(0, x)=x
$$

The functional space for the $\varphi$ variable is for $s>3 / 2$ the diffeomorphism group

$$
\mathcal{D}^{s}(\mathbb{R})=\left\{\varphi: \mathbb{R} \rightarrow \mathbb{R} \mid \varphi-\text { id } \in H^{s}(\mathbb{R}), \quad \varphi_{x}(x)>0 \text { for all } x \in \mathbb{R}\right\}
$$

where id is the identity map in $\mathbb{R}$. It is a topological group under composition of maps and consists of $C^{1}$-diffeomorphisms. For details on this space, see [1]. We can write (3) in the $\varphi$ variable as

$$
\varphi_{t t}=B\left(\varphi_{t} \circ \varphi^{-1}, \varphi_{t} \circ \varphi^{-1}\right) \circ \varphi
$$

The computations in [2] show that right side is a real analytic map

$$
\mathcal{D}^{s}(\mathbb{R}) \rightarrow P_{2}\left(H^{s}(\mathbb{R}) ; H^{s}(\mathbb{R})\right), \quad \varphi \mapsto\left[v \mapsto B\left(v \circ \varphi^{-1}, v \circ \varphi^{-1}\right) \circ \varphi\right]
$$


where we denote by $P_{2}\left(H^{s}(\mathbb{R}) ; H^{s}(\mathbb{R})\right)$ the space of continuous quadratic forms on $H^{s}(\mathbb{R})$ with values in $H^{s}(\mathbb{R})$. We can write the second-order Eq. (4) as a first-order equation on the tangent space $T \mathcal{D}^{s}(\mathbb{R})=\mathcal{D}^{s}(\mathbb{R}) \times H^{s}(\mathbb{R})$

$$
\partial_{t}\left(\begin{array}{l}
\varphi \\
v
\end{array}\right)=\left(\begin{array}{c}
v \\
B\left(v \circ \varphi^{-1}, v \circ \varphi^{-1}\right) \circ \varphi
\end{array}\right)
$$

The quadratic nature of the second component makes it to a so-called Spray—see [4]. It has in particular an exponential map. To define this map consider the ODE (5) with initial values $\varphi(0)=$ id and $v(0)=v_{0}$. Denote by $V \subseteq H^{s}(\mathbb{R})$ those initial values $v_{0}$ for which we have existence beyond time 1 . With this we define

$$
\exp : V \subseteq H^{s}(\mathbb{R}) \rightarrow \mathcal{D}^{s}(\mathbb{R}), \quad v_{0} \mapsto \varphi\left(1 ; v_{0}\right)
$$

where $\varphi\left(1 ; v_{0}\right)$ is the time 1 value of the $\varphi$-component. Because of analytic dependence on initial values exp is real analytic. Furthermore for any $v_{0} \in H^{s}(\mathbb{R})$ the curve $\varphi(t)=\exp \left(t v_{0}\right)$ is the $\varphi$-component of the solution to (5) with initial values $\varphi(0)=\mathrm{id}$ and $v(0)=v_{0}$. In particular the solution exists as long as $t v_{0} \in V$.

With this we can construct solutions to (3). So consider (3) with initial condition $v(0)=$ $v_{0} \in H^{s}(\mathbb{R})$. For $\varphi(t)=\exp \left(t v_{0}\right)$ we define

$$
v(t)=\varphi_{t}(t) \circ \varphi(t)^{-1}
$$

It turns out that $v$ is a solution to (3) - see [2], where this was established for the $b$-family of equations-and with this that $V \subseteq H^{s}(\mathbb{R})$ is the set of initial values for which (3) has a solution beyond time $T=1$. By the local well-posedness for ODEs we immediately recover the local well-posedness result of [6,7].

Theorem 2.1 The initial value problem for (1) is locally well-posed in the Sobolev spaces $H^{s}(\mathbb{R}), s>3 / 2$.

\section{Nonuniform dependence}

In this section we establish our main result Theorem 1.1. As mentioned already it will be enough to prove this for the modified Eq. (3). We can further simplify this by considering the theorem just for the time $T=1$ situation, as we have for $v$ a solution to (3) that

$$
\tilde{v}(t, x):=\lambda v(\lambda t, x)
$$

is also a solution to (3).

We proceed as in [2]. In [2] we used a conserved quantity to establish the result. For equation (3) we have something similar.

Lemma 3.1 Let $s>3 / 2$. For $v$ a solution to (3) with initial value $v(0)=v_{0} \in H^{s}(\mathbb{R})$ we have

$$
\left(\left(1-\frac{1}{\gamma^{2}} \partial_{x}^{2}\right) v(t)\right) \circ \varphi(t) \cdot \varphi_{x}(t)^{2}=\left(1-\frac{1}{\gamma^{2}} \partial_{x}^{2}\right) v_{0}+\int_{0}^{t} \frac{3 \gamma-3}{\gamma} \frac{\varphi_{t}(s) \varphi_{t x}(s)}{\varphi_{x}(s)} \mathrm{d} s
$$

where $\varphi(t)=\exp \left(t v_{0}\right)$.

The essential thing here is that the "remainder" term, the integral term which is in $H^{s-1}(\mathbb{R})$, is more regular than the first term which is in $H^{s-2}(\mathbb{R})$. 
Proof We differentiate the expression on the left in the lemma with respect to $t$ and use the equation for $v$. We have by the chain rule

$$
\begin{aligned}
\frac{d}{\mathrm{~d} t}\left(\left(\left(1-\frac{1}{\gamma^{2}} \partial_{x}^{2}\right) v\right) \circ \varphi\right) & =\left(\left(1-\frac{1}{\gamma^{2}} \partial_{x}^{2}\right) v_{t}\right) \circ \varphi+\left(\left(1-\frac{1}{\gamma^{2}} \partial_{x}^{2}\right) v_{x}\right) \circ \varphi \cdot \varphi_{t} \\
& =\left(\left(1-\frac{1}{\gamma^{2}} \partial_{x}^{2}\right) v_{t}+\left(\left(1-\frac{1}{\gamma^{2}} \partial_{x}^{2}\right) v_{x}\right) \cdot v\right) \circ \varphi \\
& =\left(\left(1-\frac{1}{\gamma^{2}} \partial_{x}^{2}\right) v_{t}+\left(1-\frac{1}{\gamma^{2}} \partial_{x}^{2}\right)\left(v_{x} \cdot v\right)+\frac{3}{\gamma^{2}} v_{x} v_{x x}\right) \circ \varphi \\
& =\left(\frac{\gamma-3}{\gamma} v v_{x}+\frac{2}{\gamma^{2}} v_{x} v_{x x}\right) \circ \varphi
\end{aligned}
$$

where we used Eq. (3) in the last equality. Therefore we have

$$
\begin{aligned}
\frac{d}{\mathrm{~d} t}\left(\left(\left(1-\frac{1}{\gamma^{2}} \partial_{x}^{2}\right) v\right) \circ \varphi \cdot \varphi_{x}^{2}\right) & =\left(v v_{x}+\frac{2}{\gamma^{2}} v_{x} v_{x x}\right) \circ \varphi \cdot \varphi_{x}^{2}+\left(v-\frac{1}{\gamma^{2}} v_{x x}\right) 2 \varphi_{x} \varphi_{t x} \\
& =\frac{3 \gamma-3}{\gamma}\left(v v_{x}\right) \circ \varphi=\frac{3 \gamma-3}{\gamma} \frac{\varphi_{t} \varphi_{t x}}{\varphi_{x}}
\end{aligned}
$$

where we used $\varphi_{t x} \varphi_{x}^{-1}=v_{x} \circ \varphi$. As $\varphi(0)=$ id integrating gives the result in the case where we work with regular solutions. But as long as $\left\|v_{x}\right\|_{L^{\infty}}$ is controlled (similar to the BealeMajda-Kato criterium) one has continuation of the solution - see [7]. Thus by approximation by regular solutions one has (6) for all $s>3 / 2$.

In the following we will use the notation

$$
y(t):=\left(1-\frac{1}{\gamma^{2}} \partial_{x}^{2}\right) v(t) \text { and } \Psi(t)=\int_{0}^{t} \frac{3 \gamma-3}{\gamma} \frac{\varphi_{t}(s) \varphi_{t x}(s)}{\varphi_{x}(s)} \mathrm{d} s
$$

Hence from (6)

$$
y(1)=\left(\frac{y(0)}{\varphi_{x}(1)^{2}}\right) \circ \varphi(1)^{-1}+\left(\frac{\Psi(1)}{\varphi_{x}(1)^{2}}\right) \circ \varphi(1)^{-1}
$$

In the following we will use also $\Psi_{v_{0}}:=\Psi(1)$ for the corresponding initial value $v_{0}$. Theorem 1.1 will follow from

Proposition 3.2 Let $V \subseteq H^{s}(\mathbb{R})$ be the domain of definition of $\exp$ (which is also the domain of definition for the time $T=1$ solution map of (3)). We denote by $v(t)$ solutions to (3). Then the map

$$
\Phi: V \subseteq H^{s}(\mathbb{R}) \rightarrow H^{s}(\mathbb{R}), \quad v(0) \mapsto v(1)
$$

is nowhere locally uniformly continuous.

To prove Proposition 3.2 we will show that $y(0) \mapsto y(1)$ is nowhere locally uniformly continuous. This is clearly enough. Before doing this we state some facts-see [2] for the proofs.

For $\varphi_{\bullet} \in \mathcal{D}^{s}(\mathbb{R})$ there is $C>0$ with

$$
\frac{1}{C}\left\|\left(\frac{y}{\widetilde{\varphi}_{x}^{2}}\right) \circ \varphi^{-1}\right\|_{s-2} \leq\|y\|_{s-2} \leq C\left\|\left(\frac{y}{\widetilde{\varphi}_{x}^{2}}\right) \circ \varphi^{-1}\right\|_{s-2}
$$

for all $y \in H^{s-2}(\mathbb{R})$ and for all $\tilde{\varphi}, \varphi$ in some neighborhood of $\varphi_{\bullet}$. 
For $\varphi_{\bullet} \in \mathcal{D}^{s}(\mathbb{R})$ there is $C>0$ with

$$
\left\|f \circ \varphi_{1}^{-1}-f \circ \varphi_{2}^{-1}\right\|_{s-2} \leq C\|f\|_{s-1}\left\|\varphi_{1}^{-1}-\varphi_{2}^{-1}\right\|_{s-2}
$$

for all $f \in H^{s-1}(\mathbb{R})$ and for all $\varphi_{1}, \varphi_{2}$ in a neighborhood of $\varphi_{\bullet}$.

Further we construct a dense subset $S \subseteq V$ with $S \subseteq H^{s+1}(\mathbb{R})$ and $d_{v} \exp \neq 0$ for all $v \in S$. Here $d_{v}$ exp is the differential of the exponential map at $v$. Take an arbitrary $v \in V \cap H^{s+1}(\mathbb{R})$ and $w \in H^{s}(\mathbb{R}), x \in \mathbb{R}$ with $w(x) \neq 0$. Consider the analytic map

$$
\mathbb{R} \rightarrow \mathbb{R}, \quad t \mapsto\left(d_{t v} \exp (w)\right)(x)
$$

which at $t=0$ is $w(x)$ (see [4] for the fact that $d_{0} \exp$ is the identity map), in particular nonzero. Thus there is a sequence $t_{n} \uparrow 1$ with $\left(d_{t_{n} v} \exp (w)\right)(x) \neq 0$. So putting $t_{n} v$ to $S$ gives the construction we need.

With this preparation we can proceed to the proof of Proposition 3.2. It is essentially the same proof as in [2] established for the $b$-family of equations.

Proof of Proposition 3.2 We take $v_{0} \in S \subseteq H^{s+1}(\mathbb{R})$ in the dense subset and show that $\Phi$ is not uniformly continuous on any ball $B_{R}\left(v_{0}\right) \subseteq V$ of radius $R>0$ with center $v_{0}$. By the construction of $S$ we can fix $g \in H^{s}(\mathbb{R})$ and $x_{0} \in \mathbb{R}$ with

$$
\left(d_{v_{0}} \exp (g)\right)\left(x_{0}\right)>m\|g\|_{s}
$$

for some $m>0$. Denote by $\varphi_{\bullet}=\exp \left(v_{0}\right)$. We choose $R_{1}>0$ in such a way that we have

$$
\frac{1}{C_{1}}\left\|\left(\frac{y}{\widetilde{\varphi}_{x}^{2}}\right) \circ \varphi^{-1}\right\|_{s-2} \leq\|y\|_{s-2} \leq C_{1}\left\|\left(\frac{y}{\widetilde{\varphi}_{x}^{2}}\right) \circ \varphi^{-1}\right\|_{s-2}
$$

for some $C_{1}>0$ for all $y \in H^{s-2}(\mathbb{R})$ and $\tilde{\varphi}, \varphi \in \exp \left(B_{R_{1}}\left(v_{0}\right)\right)$ which is possible due to the continuity properties of the composition-see [1]. Taking $0<R_{2} \leq R_{1}$ we can guarantee again by the continuity properties of the composition that

$$
\left\|y \circ \varphi^{-1}\right\|_{s-2} \leq C_{2}\|y\|_{s-2}
$$

for some $C_{2}$ and for all $y \in H^{s-2}(\mathbb{R})$ and $\varphi \in \exp \left(B_{R_{2}}\left(v_{0}\right)\right)$. Choosing $0<R_{3} \leq R_{2}$ we can ensure (see [1])

$$
\left\|f \circ \varphi_{1}^{-1}-f \circ \varphi_{2}^{-1}\right\|_{s-2} \leq \tilde{C}_{3}\|f\|_{s-1}\left\|\varphi_{1}^{-1}-\varphi_{2}^{-1}\right\|_{s-2} \leq C_{3}\|f\|_{s-1}\left\|\varphi_{1}-\varphi_{2}\right\|_{s}
$$

for some $C_{3}>0$ and for all $f \in H^{s-1}(\mathbb{R})$ and $\varphi_{1}, \varphi_{2} \in \exp \left(B_{R_{3}}\left(v_{0}\right)\right)$. Furthermore we denote by $C>0$ the constant in the Sobolev imbedding

$$
\|f\|_{L^{\infty}} \leq C\|f\|_{s}
$$

Consider the Taylor expansion for the exponential map exp : $V \rightarrow H^{s}(\mathbb{R})$

$$
\exp (w+h)=\exp (w)+d_{w} \exp (h)+\int_{0}^{1}(1-t) d_{w+t h} \exp (h, h) \mathrm{d} t
$$

We choose $0<R_{4} \leq R_{3}$ in such a way that we have

$$
\left\|d_{w}^{2} \exp \left(h_{1}, h_{2}\right)\right\|_{s} \leq K\left\|h_{1}\right\|_{s}\left\|h_{2}\right\|_{s}
$$

and

$$
\left\|d_{w_{1}}^{2} \exp \left(h_{1}, h_{2}\right)-d_{w_{2}}^{2} \exp \left(h_{1}, h_{2}\right)\right\|_{s} \leq K\left\|w_{1}-w_{2}\right\|_{s}\left\|h_{1}\right\|_{s}\left\|h_{2}\right\|_{s}
$$


for some $K>0$ and for all $w, w_{1}, w_{2} \in \exp \left(B_{R_{4}}\left(v_{0}\right)\right)$ and for all $h_{1}, h_{2} \in H^{s}(\mathbb{R})$ which is possible by the smoothness of the exponential map. By taking $0<R_{5} \leq R_{4}$ small enough we have

$$
\max \left\{C \cdot K \cdot R_{5}, C \cdot K \cdot R_{5}^{2}\right\}<m / 2
$$

By the final choice $0<R_{*} \leq R_{5}$ we can make

$$
|\varphi(x)-\varphi(y)| \leq L|x-y| \text { and }\left\|\Psi_{v}\right\|_{s} \leq M \text { and }\|\exp (v)-\exp (\tilde{v})\|_{s} \leq L\|v-\tilde{v}\|_{s}
$$

to hold for all $\varphi \in \exp \left(B_{R_{*}}\right)$ and $v, \tilde{v} \in B_{R_{*}}\left(v_{0}\right)$ due to the Sobolev imbedding and the smoothness of the exponential map. The goal is to prove that $\Phi$ is not uniformly continuous on $B_{R}\left(v_{0}\right)$ for any $0<R \leq R_{*}$. So we fix $0<R \leq R_{*}$. We define the sequence of radii

$$
r_{n}=\frac{m}{8 n}\|g\|_{s}, \quad n \geq 1
$$

and take an arbitrary smooth $w_{n}$ with support in $\left(x_{0}-\frac{r_{n}}{L}, x_{0}+\frac{r_{n}}{L}\right)$ and constant mass $\left\|w_{n}\right\|_{s}=R / 4$. Further we define $g_{n}=g / n$, which tends to zero in $H^{s}(\mathbb{R})$. With this we introduce two sequences

$$
z_{n}=v_{0}+w_{n} \text { and } \tilde{z}_{n}=z_{n}+g_{n}=v_{0}+w_{n}+g_{n}
$$

For $N$ large enough we clearly have $z_{n}, \tilde{z}_{n} \in B_{R}\left(v_{0}\right)$ for $n \geq N$ and $\left\|z_{n}-\tilde{z}_{n}\right\|_{s} \rightarrow 0$ as $n \rightarrow \infty$. Further we introduce the corresponding diffeomorphisms

$$
\varphi_{n}=\exp \left(z_{n}\right) \text { and } \tilde{\varphi}_{n}=\exp \left(\tilde{z}_{n}\right)
$$

The result will follow from $\lim \sup _{n \rightarrow \infty}\left\|\Phi\left(z_{n}\right)-\Phi\left(\tilde{z}_{n}\right)\right\|_{s}>0$. Reexpressing $\Phi$ with (6) and using the notation $y_{n}=\left(1-\frac{1}{\gamma^{2}} \partial_{x}^{2}\right) z_{n}$ and $\tilde{y}_{n}=\left(1-\frac{1}{\gamma^{2}} \partial_{x}^{2}\right) \tilde{z}_{n}$ and $\Psi_{z_{n}}, \Psi_{\tilde{z}_{n}}$ for the "remainder" terms this is equivalent to

$$
\limsup _{n \rightarrow \infty}\left\|\frac{y_{n}}{\left(\varphi_{n}\right)_{x}^{2}} \circ \varphi_{n}^{-1}-\frac{\tilde{y}_{n}}{\left(\widetilde{\varphi}_{n}\right)_{x}^{2}} \circ \widetilde{\varphi}^{-1}+\frac{\Psi_{z_{n}}}{\left(\varphi_{n}\right)_{x}^{2}} \circ \varphi_{n}^{-1}-\frac{\Psi_{\tilde{z}_{n}}}{\left(\widetilde{\varphi}_{n}\right)_{x}^{2}} \circ \widetilde{\varphi}_{n}^{-1}\right\|_{s-2}>0
$$

As the $\Psi$ terms are more regular than $H^{s-2}$, namely in $H^{s-1}$, we have

$$
\begin{aligned}
& \left\|\frac{\Psi_{z_{n}}}{\left(\varphi_{n}\right)_{x}^{2}} \circ \varphi_{n}^{-1}-\frac{\Psi_{\tilde{z}_{n}}}{\left(\widetilde{\varphi}_{n}\right)_{x}^{2}} \circ \widetilde{\varphi}_{n}^{-1}\right\|_{s-2} \\
& \leq\left\|\frac{\Psi_{z_{n}}}{\left(\varphi_{n}\right)_{x}^{2}} \circ \varphi_{n}^{-1}-\frac{\Psi_{z_{n}}}{\left(\varphi_{n}\right)_{x}^{2}} \circ \widetilde{\varphi}_{n}^{-1}\right\|_{s-2}+\left\|\frac{\Psi_{z_{n}}}{\left(\varphi_{n}\right)_{x}^{2}} \circ \widetilde{\varphi}_{n}^{-1}-\frac{\Psi_{\tilde{z}_{n}}}{\left(\widetilde{\varphi}_{n}\right)_{x}^{2}} \circ \widetilde{\varphi}_{n}^{-1}\right\|_{s-2} \\
& \leq C_{3}\left\|\frac{\Psi_{z_{n}}}{\left(\varphi_{n}\right)_{x}^{2}}\right\|_{s-1}\left\|\varphi_{n}-\widetilde{\varphi}_{n}\right\|_{s}+C_{2}\left\|\frac{\Psi_{z_{n}}}{\left(\varphi_{n}\right)_{x}^{2}}-\frac{\Psi_{\tilde{z}_{n}}}{\left(\widetilde{\varphi}_{n}\right)_{x}^{2}}\right\|_{s-2} \rightarrow 0
\end{aligned}
$$

as $n \rightarrow \infty$ since $z \mapsto \Psi_{z} /\left(\partial_{x} \exp (z)\right)^{2}$ is smooth. Thus it remains to establish

$$
\limsup _{n \rightarrow \infty}\left\|\frac{y_{n}}{\left(\varphi_{n}\right)_{x}^{2}} \circ \varphi_{n}^{-1}-\frac{\tilde{y}_{n}}{\left(\widetilde{\varphi}_{n}\right)_{x}^{2}} \circ \widetilde{\varphi}_{n}^{-1}\right\|_{s-2}>0
$$

We split

$$
y_{n}=\left(1-\frac{1}{\gamma^{2}} \partial_{x}^{2}\right)\left(v_{0}+w_{n}\right) \text { resp. } \tilde{y}_{n}=\left(1-\frac{1}{\gamma^{2}} \partial_{x}^{2}\right)\left(v_{0}+w_{n}+g_{n}\right)
$$


As $v_{0} \in H^{s+1}$ we can treat the $v_{0}$ terms in the same way as the $\Psi$ terms and get

$$
\lim _{n \rightarrow \infty}\left\|\frac{\left(1-\frac{1}{\gamma^{2}} \partial_{x}^{2}\right) v_{0}}{\left(\varphi_{n}\right)_{x}^{2}} \circ \varphi_{n}^{-1}-\frac{\left(1-\frac{1}{\gamma^{2}} \partial_{x}^{2}\right) v_{0}}{\left(\widetilde{\varphi}_{n}\right)_{x}^{2}} \circ \widetilde{\varphi}_{n}^{-1}\right\|_{s-2}=0
$$

For the $g_{n}$ term we have trivially

$$
\left\|\frac{\left(1-\frac{1}{\gamma^{2}} \partial_{x}^{2}\right) g_{n}}{\left(\widetilde{\varphi}_{n}\right)_{x}^{2}} \circ \widetilde{\varphi}_{n}^{-1}\right\|_{s-2} \leq C_{1}\left\|\left(1-\frac{1}{\gamma^{2}} \partial_{x}^{2}\right) g_{n}\right\|_{s-2} \rightarrow 0
$$

The only remaining thing is to consider

$$
\limsup _{n \rightarrow \infty}\left\|\frac{\left(1-\frac{1}{\gamma^{2}} \partial_{x}^{2}\right) w_{n}}{\left(\varphi_{n}\right)_{x}^{2}} \circ \varphi_{n}^{-1}-\frac{\left(1-\frac{1}{\gamma^{2}} \partial_{x}^{2}\right) w_{n}}{\left(\widetilde{\varphi}_{n}\right)_{x}^{2}} \circ \widetilde{\varphi}_{n}^{-1}\right\|_{s-2}
$$

In order to estimate this from below we will establish that the two terms have disjoint support. This we do by estimating the distance $\left|\varphi_{n}\left(x_{0}\right)-\widetilde{\varphi}_{n}\left(x_{0}\right)\right|$. By the Taylor expansion we have

$$
\varphi_{n}=\exp \left(v_{0}\right)+d_{v_{0}} \exp \left(w_{n}\right)+\int_{0}^{1}(1-t) d_{v_{0}+t w_{n}}^{2} \exp \left(w_{n}, w_{n}\right) \mathrm{d} t
$$

resp.

$$
\widetilde{\varphi}_{n}=\exp \left(v_{0}\right)+d_{v_{0}} \exp \left(w_{n}+g_{n}\right)+\int_{0}^{1}(1-t) d_{v_{0}+t\left(w_{n}+g_{n}\right)}^{2} \exp \left(w_{n}+g_{n}, w_{n}+g_{n}\right) \mathrm{d} t
$$

Taking the difference we can write

$$
\tilde{\varphi}_{n}-\varphi_{n}=d_{v_{0}} \exp \left(g_{n}\right)+\mathcal{R}_{1}+\mathcal{R}_{2}+\mathcal{R}_{3}
$$

where

$$
\mathcal{R}_{1}=\int_{0}^{1}(1-t)\left(d_{v_{0}+t\left(w_{n}+g_{n}\right)}^{2}\left(w_{n}, w_{n}\right)-d_{v_{0}+t w_{n}}^{2}\left(w_{n}, w_{n}\right)\right) \mathrm{d} t
$$

and

$$
\mathcal{R}_{2}=\int_{0}^{1}(1-t) d_{v_{0}+t\left(w_{n}+g_{n}\right)}^{2}\left(g_{n}, g_{n}\right) \mathrm{d} t
$$

and

$$
\mathcal{R}_{2}=2 \int_{0}^{1}(1-t) d_{v_{0}+t\left(w_{n}+g_{n}\right)}^{2}\left(w_{n}, g_{n}\right) \mathrm{d} t
$$

For these we have

$$
\left\|\mathcal{R}_{1}\right\|_{\infty} \leq C\left\|\mathcal{R}_{1}\right\|_{s} \leq C K\left\|g_{n}\right\|_{s}\left\|w_{n}\right\|_{s}^{2} \leq \frac{1}{n} C K\|g\|_{s}(R / 4)^{2} \leq \frac{1}{4 n} C K R^{2}\|g\|_{s}
$$

and

$$
\left\|\mathcal{R}_{2}\right\|_{\infty} \leq C\left\|\mathcal{R}_{2}\right\|_{s} \leq 2 C K\left\|g_{n}\right\|_{s}\left\|w_{n}\right\|_{s} \leq \frac{1}{n} C K\|g\|_{s}(R / 4) \leq \frac{2}{4 n} C K R\|g\|_{s}
$$

and

$$
\left\|\mathcal{R}_{3}\right\|_{\infty} \leq C\left\|\mathcal{R}_{3}\right\|_{s} \leq C K\left\|g_{n}\right\|_{s}^{2} \leq \frac{1}{n} C K\|g\|_{s}(R / 4) \leq \frac{1}{4 n} C K R\|g\|_{s}
$$


Therefore

$$
\begin{aligned}
\left|\varphi_{n}\left(x_{0}\right)-\widetilde{\varphi}_{n}\left(x_{0}\right)\right| & \geq\left|d_{v_{0}} \exp \left(g_{n}\right)\right|-\left\|\mathcal{R}_{1}\right\|_{\infty}-\left\|\mathcal{R}_{2}\right\|_{\infty}-\left\|\mathcal{R}_{3}\right\|_{\infty} \\
& \geq \frac{1}{n} m\|g\|_{s}-\frac{1}{n} \frac{m}{2}\|g\|_{s}=\frac{m}{2 n}\|g\|_{s}
\end{aligned}
$$

The support of $\frac{\left(1-\frac{1}{\gamma^{2}} \partial_{x}^{2}\right) w_{n}}{\left(\varphi_{n}\right)_{x}^{2}} \circ \varphi_{n}^{-1}$ is contained in $\left(\varphi_{n}\left(x_{0}\right)-r_{n}, \varphi_{n}\left(x_{0}\right)+r_{n}\right)$ taking into account the Lipschitz property of $\varphi_{n}$ with Lipschitz constant $L$ and the definition of $w_{n}$. Analogously the support of $\frac{\left(1-\frac{1}{\gamma^{2}} \partial_{x}^{2}\right) w_{n}}{\left(\widetilde{\varphi}_{n}\right)_{x}^{2}} \circ \widetilde{\varphi}_{n}^{-1}$ is contained in $\left(\widetilde{\varphi}_{n}\left(x_{0}\right)-r_{n}, \widetilde{\varphi}_{n}\left(x_{0}\right)+r_{n}\right)$. As we have

$$
r_{n} \leq\left|\varphi_{n}\left(x_{0}\right)-\tilde{\varphi}_{n}\left(x_{0}\right)\right| / 4
$$

we can "separate" the disjointly supported terms (see also [2]). Thus we have

$$
\begin{aligned}
& \limsup _{n \rightarrow \infty}\left\|\frac{\left(1-\frac{1}{\gamma^{2}} \partial_{x}^{2}\right) w_{n}}{\left(\varphi_{n}\right)_{x}^{2}} \circ \varphi_{n}^{-1}-\frac{\left(1-\frac{1}{\gamma^{2}} \partial_{x}^{2}\right) w_{n}}{\left(\widetilde{\varphi}_{n}\right)_{x}^{2}} \circ \widetilde{\varphi}_{n}^{-1}\right\|_{s-2}^{2} \\
& \geq \limsup _{n \rightarrow \infty} \tilde{C}\left(\left\|\frac{\left(1-\frac{1}{\gamma^{2}} \partial_{x}^{2}\right) w_{n}}{\left(\varphi_{n}\right)_{x}^{2}} \circ \varphi_{n}^{-1}\right\|_{s-2}^{2}+\left\|\frac{\left(1-\frac{1}{\gamma^{2}} \partial_{x}^{2}\right) w_{n}}{\left(\widetilde{\varphi}_{n}\right)_{x}^{2}} \circ \tilde{\varphi}_{n}^{-1}\right\|_{s-2}^{2}\right) \\
& \geq \limsup _{n \rightarrow \infty} \tilde{C} \frac{2}{C^{2}}\left\|\left(1-\frac{1}{\gamma^{2}} \partial_{x}^{2}\right) w_{n}\right\|_{s-2}^{2} \geq \limsup _{n \rightarrow \infty} \tilde{K}\left\|w_{n}\right\|_{s}^{2}=\tilde{K} R^{2} / 4
\end{aligned}
$$

So for any $R \leq R_{*}$ we have constructed $\left(z_{n}\right)_{n \geq N},\left(\tilde{z}_{n}\right)_{n \geq N} \subseteq B_{R}\left(u_{0}\right)$ with $\lim _{n \rightarrow \infty} \| z_{n}-$ $\tilde{z}_{n} \|_{s}=0$ and $\lim \sup _{n \rightarrow \infty}\left\|\Phi\left(z_{n}\right)-\Phi\left(\tilde{z}_{n}\right)\right\|_{s} \geq C \cdot R$ for some constant $C>0$ independent of $R$ showing the claim.

\section{References}

1. Inci, H., Kappeler, T., Topalov, P.: On the regularity of the composition of diffeomorphisms. Mem. Am. Math. Soc. 226(1062), 45 (2013)

2. Inci, H.: On the well-posedness of the Holm-Staley $b$-family of equations. J. Nonlinear Math. Phys. 23(2), 213-233 (2016)

3. Karapetyan, D.: Non-uniform dependence and well-posedness for the hyperelastic rod equation. J. Differ. Equ. 249(4), 796-826 (2010)

4. Lang, S.: Differential and Riemannian Manifolds, 3rd edn. Springer, New York (1995)

5. Olson, E.: Non-uniform dependence on initial data for a family of non-linear evolution equations. Differ. Integral Equ. 19(10), 10811102 (2006)

6. Yin, Z.: On the Cauchy problem for a nonlinearly dispersive wave equation. J. Nonlinear Math. Phys. 10(1), 10-15 (2003)

7. Zhou, Y.: Local well-posedness and blow-up criteria of solutions for a rod equation. Math. Nachr. 278(14), 749-777 (2005) 